\title{
Immunophenotyping in systemic mastocytosis diagnosis: 'CD25 positive' alone is more informative than the 'CD25 and/or CD2' WHO criterion
}

\author{
José Mário T Morgado ${ }^{1,2,4}$, Laura Sánchez-Muñoz ${ }^{1,2,4}$, Cristina G Teodósio ${ }^{2,3}$, \\ Maria Jara-Acevedo ${ }^{2,3}$, Iván Álvarez-Twose ${ }^{1,2}$, Almudena Matito ${ }^{1,2}$, \\ Elisa Fernández-Nuñez ${ }^{2,3}$, Andrés García-Montero ${ }^{2,3}$, Alberto Orfao ${ }^{2,3,4}$ \\ and Luís Escribano ${ }^{1,2,4}$
}

${ }^{1}$ Instituto de Estudios de Mastocitosis de Castilla La Mancha, Hospital Virgen del Valle, Toledo, Spain;
${ }^{2}$ Spanish Network on Mastocytosis (REMA), Spain and ${ }^{3}$ Centro de Investigación del Cáncer/IBMCC,
Departamento de Medicina and Servicio General de Citometría, University of Salamanca, Salamanca, Spain

\begin{abstract}
Aberrant expression of CD2 and/or CD25 by bone marrow, peripheral blood or other extracutaneous tissue mast cells is currently used as a minor World Health Organization diagnostic criterion for systemic mastocytosis. However, the diagnostic utility of CD2 versus CD25 expression by mast cells has not been prospectively evaluated in a large series of systemic mastocytosis. Here we evaluate the sensitivity and specificity of CD2 versus $\mathrm{CD} 25$ expression in the diagnosis of systemic mastocytosis. Mast cells from a total of $\mathbf{8 8 6}$ bone marrow and 153 other non-bone marrow extracutaneous tissue samples were analysed by multiparameter flow cytometry following the guidelines of the Spanish Network on Mastocytosis at two different laboratories. The 'CD25 + and/or CD2 + bone marrow mast cells' World Health Organization criterion showed an overall sensitivity of $100 \%$ with $99.0 \%$ specificity for the diagnosis of systemic mastocytosis whereas CD25 expression alone presented a similar sensitivity (100\%) with a slightly higher specificity (99.2\%). Inclusion of CD2 did not improve the sensitivity of the test and it decreased its specificity. In tissues other than bone marrow, the mast cell phenotypic criterion revealed to be less sensitive. In summary, CD2 expression does not contribute to improve the diagnosis of systemic mastocytosis when compared with aberrant CD25 expression alone, which supports the need to update and replace the minor World Health Organization 'CD25 + and/or CD2 +' mast cell phenotypic diagnostic criterion by a major criterion based exclusively on CD25 expression.
\end{abstract}

Modern Pathology (2012) 25, 516-521; doi:10.1038/modpathol.2011.192; published online 6 January 2012

Keywords: CD2; CD25; diagnostic criteria; immunophenotype; systemic mastocytosis

Mastocytosis includes a heterogeneous group of disorders characterised by the presence of abnormal mast cells in one or multiple organs such as the skin or bone marrow, among others. ${ }^{1}$ Bone marrow biopsy and aspirate studies are essential for precise diagnosis and classification of the disease, ${ }^{2}$ eg,

Correspondence: Dr L Escribano, MD, PhD, Instituto de Estudios de Mastocitosis de Castilla La Mancha, Hospital Virgen del Valle, Toledo, Spain.

E-mail: lescribanom@sescam.jccm.es

${ }^{4}$ These authors contributed equally to this work.

Received 29 August 2011; revised 28 September 2011; accepted 28 September 2011; published online 6 January 2012 differential diagnosis between cutaneous mastocytosis and systemic mastocytosis. A decade ago, a consensus classification of mastocytosis was proposed, which was adopted later on by the World Health Organization. ${ }^{1-3}$ In addition to a major conventional histopathological criterion (multifocal dense aggregates of $\geq 15$ mast cells in bone marrow and/or other extracutaneous tissues), four minor morphological (atypical mast cells in smears or biopsy sections of bone marrow or other extracutaneous organs), immunophenotypical (CD25 + and/ or CD2 + mast cells), molecular (D816V KIT mutation) and biochemical (serum tryptase levels persistently $>20 \mathrm{ng} / \mathrm{ml}$ ) criteria are proposed in this 
classification for the diagnosis of systemic mastocytosis. In recent years, major methodologicalrelated advances have been made regarding the diagnosis of tissue involvement in mastocytosis. These have contributed to a more sensitive and precise evaluation of the immunophenotype of mast cells by both immunohistochemistry ${ }^{4-6}$ and flow cytometry, ${ }^{7-10}$ and the identification of KIT mutations; ${ }^{11,12}$ such advances are particularly relevant at the early stages of the disease, eg among indolent systemic mastocytosis patients who display very low mast cell burden, in the absence of bone marrow mast cell aggregates.

Early studies about the immunophenotype of bone marrow mast cells have shown aberrant coexpression of CD2 and CD25 in indolent systemic mastocytosis patients versus bone marrow samples from healthy subjects and patients suffering from different haematological and non-haematological disease conditions other than mastocytosis ${ }^{8,13}$ who systematically showed CD25-/CD2- bone marrow mast cells. Subsequent studies reported variable frequencies of CD2-negative mastocytosis cases, suggesting that CD2 expression could be less prevalent than initially suspected, its frequency also depending on the sensitivity of the reagent used for its evaluation. Accordingly, while a frequency of between 30 and $63 \%$ CD2 + cases was found with FITC-conjugated reagents, this would increase to up to $92 \%$ when a more sensitive PE-conjugated antibody reagent was used..$^{9,14}$

With this variability in mind, the World Health Organization recommended that the expression of CD2 and/or CD25 by mast cells from bone marrow, peripheral blood or other extracutaneous tissues should be a minor phenotypic criterion for systemic mastocytosis. However, to the best of our knowledge, so far no studies have been reported in which the specificity and sensitivity of the two phenotypic markers have been prospectively analysed in a large number of systemic mastocytosis patients versus controls. Here, we examine the expression profile of CD2 and CD25 antigens in a large cohort of bone marrow samples and non-bone marrow extracutaneous specimens in order to retrospectively evaluate the utility of both markers once individually considered versus the combined 'CD25 + and/or CD2 +' World Health Organization phenotypic criterion in the diagnostic work-up of systemic mastocytosis.

\section{Materials and methods}

\section{Patients, Controls and Samples}

All samples were obtained at the Instituto de Mastocitosis de Castilla La Mancha (Toledo, Spain), and, to be included in the study, the recommendations and requirements of the local Ethics Committee were strictly followed after the study was approved by the Institution Review Board.
According to the World Health Organization criteria, ${ }^{2,3} 276$ patients were diagnosed with systemic mastocytosis. Most patients were classified as indolent systemic mastocytosis with skin lesions $(n=196)$ and indolent systemic mastocytosis without skin lesions $(n=56) ; 16$ cases corresponded to aggressive systemic mastocytosis, 6 to indolent systemic mastocytosis with an associated clonal haematological non-mast cell lineage disease and 2 to aggressive systemic mastocytosis with an associated clonal haematological non-mast cell lineage disease. From these patients, a total of 276 bone marrow samples and 27 extracutaneous tissue samples were analysed. The other 610 bone marrow samples and 126 extracutaneous tissue samples corresponded to patients suffering from different haematological and non-haematological disorders other than mastocytosis, whose specimens were studied either for diagnostic purposes or for follow-up of previously diagnosed diseases. In addition to non-mast cell-related diseases, this later group included 51 cases diagnosed as clonal $(N=11)$ and non-clonal $(N=37)$ mast cells activation syndromes and mastocytomas $(N=3)$ who did not fulfilled the criteria for systemic mastocytosis.

\section{Immunophenotypic Studies}

Immunophenotypic analysis of bone marrow mast cells was performed by multiparameter flow cytometry following the Spanish Network on Mastocytosis guidelines. ${ }^{9,10}$ Briefly, bone marrow samples were passed several times through a 25-gauge needle to disaggregate the bone marrow particles and stained with fluorescent-labelled monoclonal antibodies specific for CD117, CD45, CD25 and CD2. In a first acquisition step, $5 \times 10^{4}$ events per tube, corresponding to the lysed whole bone marrow sample, were collected and information on them stored. A second acquisition of the cells present in the same tube was performed for at least $3 \times 10^{5}$ events/tube but only those included in a preestablished SSC/CD117 gate were stored to increase the sensitivity of the method (Figure 1). Mast cells were identified by their high expression of CD117, intermediate expression of CD45 and variable lightscattering characteristics (Figure 1). Both external and internal quality controls were routinely applied and samples were studied in parallel at two different laboratories of the Spanish Network on Mastocytosis, as described elsewhere. ${ }^{9,10}$ An antigen was considered to be positive when its mean fluorescence intensity exceeded the mean fluorescence intensity plus 3 standard deviations of the mean of the corresponding baseline autofluorescence levels measured for unstained cells. Sensitivity, specificity, test efficiency and both positive and negative predictive values, were calculated as follows: sensitivity $=$ number of true positives/ (number of true positives + number of false 


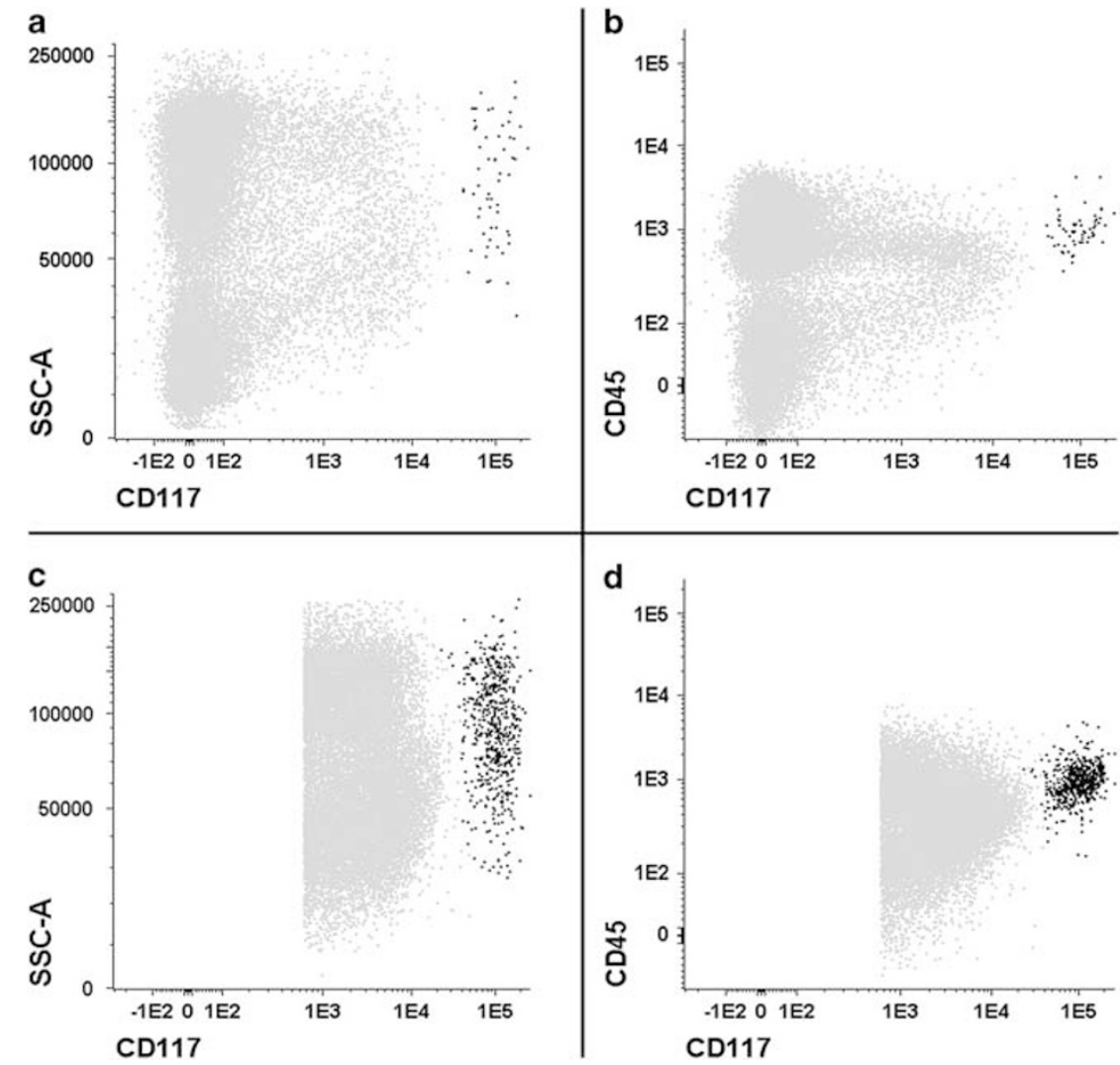

Figure 1 Mast cell identification by flow cytometry. In a first acquisition step $5 \times 10^{4}$ events/tube were acquired and data on them stored $(\mathbf{a}, \mathbf{b})$. In a second acquisition step at least $3 \times 10^{5}$ events/tube were acquired but only those included in a pre-established SSC/CD117 gate were stored to increase the sensitivity of the method $(\mathbf{c}, \mathbf{d})$.

negatives); specificity $=$ number of true negatives/ (number of true negatives + number of false positives); test efficiency $=$ (number of true positives + number of true negatives)/(number of true positives + number of true negatives + number of false positives + number of false negatives); positive predictive value $=$ number of true positives/(number of true positives + number of false positives); negative predictive value $=$ number of true negatives/(number of true negatives + number of false negatives). The $\chi^{2}$ test was applied to evaluate the statistical significance of differences between groups.

\section{Results}

CD25 was systematically expressed by bone marrow mast cells from 276/276 systemic mastocytosis cases (sensitivity of $100 \%$ ), but only in a minority $(n=5 / 610)$ of bone marrow mast cells from cases without systemic mastocytosis (specificity of 99. $2 \%$; Table 1) which corresponded to one patient with hypereosinophilia and the FIPL1-PDGFRA fusion gene and four clonal mast cell activation syndromes. In turn, CD2 was positive in bone marrow mast cells from most $(n=230 / 276)$ systemic mastocytosis bone marrow samples (sensitivity of $83.3 \%$ ) and in 6/610 non-systemic mastocytosis bone marrow samples (specificity of $99.2 \%$; Table 1). As shown in Table 2, aggressive systemic mastocytosis patients included a higher fraction of cases with CD25 +/CD2- mast cells in their bone marrow $(28 \%)$ when compared with indolent systemic mastocytosis with $(15 \%)$ and without $(18 \%)$ skin lesions. In extracutaneous tissues other than bone marrow, the sensitivity and specificity observed for CD25 $(77.8 \%$ and $96.8 \%$, respectively) and for CD2 (40.7\% and $85.7 \%$, respectively) were considerably lower than observed in bone marrow (Table 1) and, as described above for bone marrow samples, the frequency of cases with CD25 + /CD2mast cells was higher among the aggressive systemic mastocytosis when compared with indolent systemic mastocytosis with and without skin lesions cases (53 vs 22 and $0 \%$, respectively).

Based on these results, CD25 alone showed a higher efficiency than CD2 alone and the 'CD25+ and/or CD2 +' World Health Organization criterion for bone marrow (99.4\% versus 94.2 and $99.3 \%$, respectively) and particularly for other extracutaneous non-bone marrow tissues (93.5\% versus 77.8 and $83.0 \%$, respectively).

Noteworthy, among patients without mastocytosis, cases with clonal (KIT D816V mutation-positive) 
Table 1 Sensitivity, specificity and both negative and positive predictive values of CD25 and CD2 expression on mast cells from bone marrow and non-bone marrow extracutaneous tissues

\begin{tabular}{|c|c|c|c|c|c|c|}
\hline & \multicolumn{3}{|c|}{ Bone marrow mast cells } & \multicolumn{3}{|c|}{ Non-bone marrow extracutaneous tissues mast cells } \\
\hline & $C D 25+$ & $C D 2+$ & CD25+ and/or CD2+ & $C D 25+$ & $C D 2+$ & CD25+ and/or $C D 2+$ \\
\hline $\begin{array}{l}\text { Sensitivity (\%) } \\
(95 \% \text { CI, \%) }\end{array}$ & $\begin{array}{c}100 \\
(98-100)\end{array}$ & $\begin{array}{c}83.3 \\
(78-87)\end{array}$ & $\begin{array}{c}100 \\
(98-100)\end{array}$ & $\begin{array}{c}77.8 \\
(57-91)\end{array}$ & $\begin{array}{c}40.7 \\
(23-61)\end{array}$ & $\begin{array}{c}81.5 \\
(61-91)\end{array}$ \\
\hline $\begin{array}{l}\text { Specificity (\%) } \\
(95 \% \text { CI, \%) }\end{array}$ & $\begin{array}{c}99.2 \\
(98-100)\end{array}$ & $\begin{array}{c}99.2 \\
(98-100)\end{array}$ & $\begin{array}{c}99.0 \\
(98-100)\end{array}$ & $\begin{array}{c}96.8 \\
(92-99)\end{array}$ & $\begin{array}{c}85.7 \\
(78-91)\end{array}$ & $\begin{array}{c}83.3 \\
(75-89)\end{array}$ \\
\hline $\begin{array}{l}\text { Positive PV (\%) } \\
(95 \% \text { CI, \%) }\end{array}$ & $\begin{array}{c}98.2 \\
(96-99\end{array}$ & $\begin{array}{c}97.9 \\
(95-99)\end{array}$ & $\begin{array}{c}97.9 \\
(95-99)\end{array}$ & $\begin{array}{c}84.0 \\
(63-95)\end{array}$ & $\begin{array}{c}37.9 \\
(21-58)\end{array}$ & $\begin{array}{c}51.2 \\
(36-66)\end{array}$ \\
\hline $\begin{array}{l}\text { Negative PV (\%) } \\
(95 \% \text { CI, \%) }\end{array}$ & $\begin{array}{c}100 \\
(99-100)\end{array}$ & $\begin{array}{c}92.9 \\
(91-95)\end{array}$ & $\begin{array}{c}100 \\
(99-100)\end{array}$ & $\begin{array}{c}95.3 \\
(90-98)\end{array}$ & $\begin{array}{c}87.1 \\
(80-92)\end{array}$ & $\begin{array}{c}95.5 \\
(89-98)\end{array}$ \\
\hline $\begin{array}{l}\text { Efficiency (\%) } \\
(95 \% \text { CI, \%) }\end{array}$ & $\begin{array}{c}99.4 \\
(99-100)\end{array}$ & $\begin{array}{c}94.2 \\
(93-96)\end{array}$ & $\begin{array}{c}99.3 \\
(99-100)\end{array}$ & $\begin{array}{c}93.5 \\
(88-96)\end{array}$ & $\begin{array}{c}77.8 \\
(71-84)\end{array}$ & $\begin{array}{c}83.0 \\
(76-88)\end{array}$ \\
\hline
\end{tabular}

PV: predictive value.

Table 2 Association between presence/absence of CD2 and subtype of disease

\begin{tabular}{|c|c|c|c|c|c|c|c|c|}
\hline & \multicolumn{4}{|c|}{ Bone marrow mast cells } & \multicolumn{4}{|c|}{ Non-bone marrow extracutaneous tissues mast cells } \\
\hline & $\begin{array}{l}\text { ISMs- } \\
(\mathrm{N}=56)\end{array}$ & $\begin{array}{c}{I S M S+^{\mathrm{a}}} \\
(\mathrm{N}=202)\end{array}$ & $\begin{array}{c}A S M^{\mathrm{b}} \\
(\mathrm{N}=18)\end{array}$ & $\begin{array}{c}\chi^{2} \\
\text { (P-value) }\end{array}$ & $\begin{array}{l}\text { ISMs- } \\
(\mathrm{N}=1)\end{array}$ & $\begin{array}{l}I S M s+^{\mathrm{a}} \\
(\mathrm{N}=9)\end{array}$ & $\begin{array}{c}A S M^{\mathrm{b}} \\
(\mathrm{N}=17)\end{array}$ & $\begin{array}{c}\chi^{2} \\
\text { (P-value) }\end{array}$ \\
\hline $\mathrm{CD} 25+/ \mathrm{CD} 2-$ & $10 / 56(18 \%)$ & $31 / 202(15 \%)$ & $5 / 18(28 \%)$ & $1.33(0.51)$ & $0 / 1(0 \%)$ & $2 / 9(22 \%)$ & $9 / 17(53 \%)$ & $1.82(0.40)$ \\
\hline CD25+/CD2+ & $46 / 56(82 \%)$ & $171 / 202(85 \%)$ & $13 / 18(72 \%)$ & $0.32(0.85)$ & $0 / 1(0 \%)$ & $3 / 9(33 \%)$ & $7 / 17(41 \%)$ & $0.51(0.78)$ \\
\hline
\end{tabular}

ISMs-: indolent systemic mastocytosis without skin lesions; ISMs+: indolent systemic mastocytosis with skin lesions; ASM: aggressive systemic mastocytosis.

${ }^{\mathrm{a}}$ Includes ISM associated with haematological non-mast cell related disease (AHNMD).

${ }^{\mathrm{b}}$ Includes ASM-AHNMD.

mast cells were included who did not fulfil the criteria for systemic mastocytosis and that were thus classified as clonal systemic mast cell activation syndromes $(n=11)$. Four of these patients presented, at diagnosis, CD25 +/CD2 + mast cells in their bone marrow, which also carried the D816V KIT mutation in the absence of other criteria for systemic mastocytosis.

The different patterns of expression of CD25 and CD2 observed on bone marrow and non-bone marrow extracutaneous tissue mast cells from systemic mastocytosis and from cases without systemic mastocytosis (CD25-/CD2-, CD25 + / CD2-, CD25-/CD2 + and CD25 + /CD2 + ) are illustrated in Figure 2. Of note, individual patients could show a single or a mixture of two or three phenotypically distinct mast cell populations. Moreover, all systemic mastocytosis patients showed CD25 + bone marrow mast cells and a significant percentage of CD25 +/CD2- cases was observed.

\section{Discussion}

Early studies about the immunophenotype of bone marrow mast cells in systemic mastocytosis reported occurrence of multiple aberrant phenotypes that allowed for their clear-cut discrimination from both normal and reactive bone marrow mast cells..$^{8,9,13,15,16}$ Among other markers, aberrant expression of CD2 and CD25 was highlighted as a potential hallmark of systemic mastocytosis, as both proteins were absent in normal/reactive bone marrow mast cells while coexpressed in the great majority of systemic mastocytosis patients. ${ }^{8,13,17}$ Further studies confirmed these findings and at the same time they showed that among the two markers, CD25 could be more sensitive and reliable than CD2 as positivity for the later marker largely depends on the sensitivity of the fluorochromeconjugated antibody reagent used with sensitivity rates of $67 \%$ and $87 \%$ for CD2-FITC and CD2-PE reagents, respectively. ${ }^{9}$ These results indicate that 


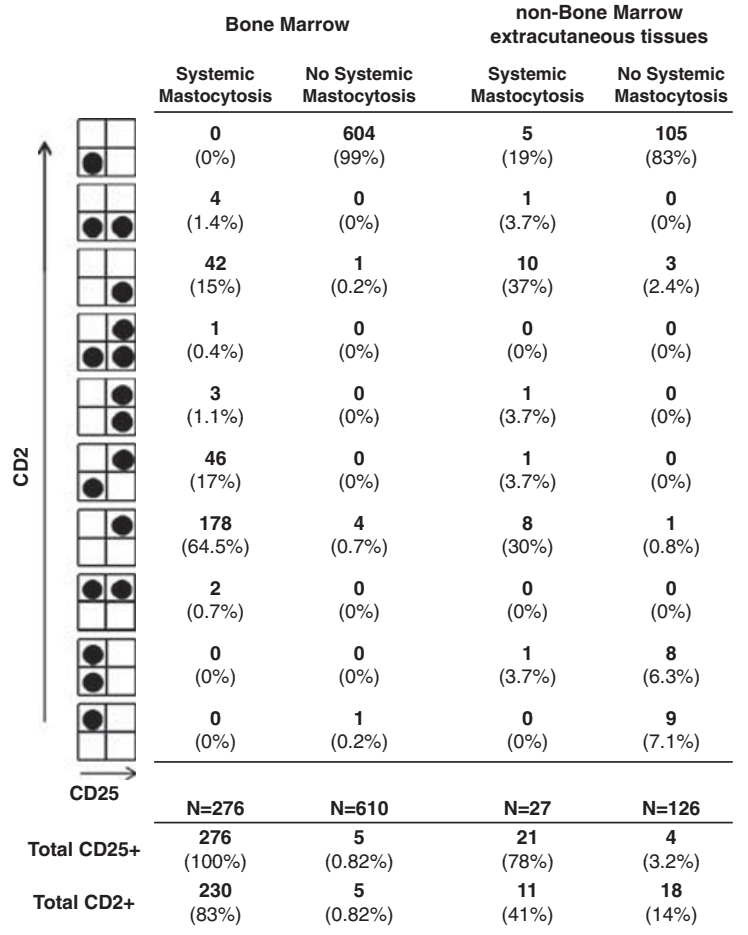

Figure 2 Frequency of different MC phenotypic profiles observed in SM versus non-SM cases as defined by CD2 and CD25 expression. Phenotypes are shown as schematic bivariate CD25 versus CD2 plots with MC populations represented as black circles. Combined phenotypes constituted by two or more phenotypically distinct MC populations are represented as multiple circles in the same plot.

the panel of antibodies and fluorochrome-conjugated reagents selected is critical for adequate flow cytometry evaluation of the phenotype of mast cells.

Interestingly, all systemic mastocytosis patients showed CD25 + bone marrow mast cells, including a significant percentage of CD25 + /CD2- cases, while only one of 610 cases without systemic mastocytosis being CD25+, if we exclude a few clonal mast cell activation syndrome cases. Conversely, no CD25-/CD2 + systemic mastocytosis cases were detected. Overall, the sensitivity of CD25 alone was the same as that of the World Health Organization criterion with a slightly greater specificity. Altogether, these results indicate that in the diagnosis of bone marrow involvement in systemic mastocytosis, the value of CD2 expression assessment does not add to that of CD25 alone. Interestingly, similar results were observed when the expression of CD2 and CD25 was evaluated on mast cells from other extracutaneous tissues, with a greater efficiency for CD25 versus CD2, this later marker being of no added value. Despite this, the two markers also showed a significantly lower sensitivity when evaluated on mast cells from extracutaneous tissues other than bone marrow versus bone marrow. In fact, the sensitivity and specificity of both CD2 and CD25, and therefore their diagnostic value, were significantly lower when assessed in tissues other than bone marrow. For this reason, a bone marrow study should be made whenever possible and may be sufficient to the diagnosis of systemic mastocytosis. Beyond the higher sensitivity of mast cell immunophenotyping of bone marrow versus other extracutaneous tissue samples, the former also further allows purification of enough cells from different haematopoietic cell compartments, to assess multilineage involvement by the KIT mutation, which has proven to be the most powerful independent prognostic factor for progression of indolent systemic mastocytosis cases to more aggressive forms of the disease and other malignant myeloid disorders. ${ }^{18}$

As mentioned above, among the cases without systemic mastocytosis, few patients classified as clonal mast cell activation syndromes were included; interestingly, 4/11 clonal mast cell activation syndrome cases presented CD25 + bone marrow mast cells, which significantly contributed to decrease the specificity of the phenotypic criterion in our series. Criteria like the presence of mast cell aggregates and serum tryptase levels are less likely to be fulfilled in patients with low mast cell burden and the possibility that clonal mast cell activation syndromes with phenotypically aberrant bone marrow mast cells may correspond to true systemic mastocytosis cases was already discussed elsewhere, ${ }^{19}$ pointing out an even potentially greater specificity of the bone marrow mast cell phenotype in the diagnosis of systemic mastocytosis, than that reported here.

In our routine workflow, we assess CD25 expression by both flow cytometry in bone marrow aspirates and immunohistochemistry in bone marrow biopsies. As reported by other groups, ${ }^{6,20}$ the assessment of CD25 expression in neoplastic mast cells by immunohistochemistry is of great diagnostic value. Nevertheless, in our experience, this is true in cases having compact mast cell aggregates or diffuse, loosely scattered mast cells, but not in cases with low bone marrow mast cell numbers such as patients with indolent systemic mastocytosis at the early stages of the disease lacking compact mast cell aggregates representing around $20 \%$ in patients with skin lesions ${ }^{18,19,21}$ and around $37 \%$ in patients lacking skin lesions. ${ }^{19,21}$ In such cases, the assessment of CD25 expression in neoplastic mast cells by immunohistochemistry is neither completely sensitive nor specific for distinguishing CD25 + mast cells from other CD25expressing cells such as activated T-lymphocytes. ${ }^{22}$ We believe that both techniques complement each other and both should be employed whenever possible.

In summary, our results suggest that expression of CD2 does not add to that of CD25 in the diagnosis of systemic mastocytosis, and that this should be considered in future revisions of the current 
World Health Organization phenotypic criterion for systemic mastocytosis.

\section{Acknowledgement}

This work was supported by Grants from the Fondo de Investigaciones Sanitarias (FIS) of the Ministerio de Ciencia e Innovación of Spain (PS09/00032); Fundación Sociosanitaria de Castilla-La Mancha (2007/36, 2010/008 and G-2010/C-002).

\section{Disclosure/conflict of interest}

The authors declare no conflict of interest.

\section{References}

1 Valent P, Horny HP, Escribano L, et al. Diagnostic criteria and classification of mastocytosis: a consensus proposal. Leuk Res 2001;25:603-625.

2 Valent P, Horny HP, Li CY, et al. Mastocytosis (mast cell disease). World Health Organization (WHO) Classification of Tumours. Pathology \& Genetics. Tumours of Haematopoietic and Lymphoid Tissues. IARC Press: Lyon, France, 2001;291-302.

3 Horny HP, Metcalfe DD, Bennet JM, et al. Mastocytosis. WHO Classification of Tumours of Haematopoietic and Lymphoid Tissues, 4th edn. IARC: Lyon, France, 2008. pp 54-63.

4 Horny HP, Sillaber C, Menke D, et al. Diagnostic value of immunostaining for tryptase in patients with mastocytosis. Am J Surg Pathol 1998;22:1132-1140.

5 Sotlar K, Horny HP, Simonitsch I, et al. CD25 indicates the neoplastic phenotype of mast cells: a novel immunohistochemical marker for the diagnosis of systemic mastocytosis (SM) in routinely processed bone marrow biopsy specimens. Am J Surg Pathol 2004;28:1319-1325.

6 Krokowski M, Sotlar K, Krauth MT, et al. Delineation of patterns of bone marrow mast cell infiltration in systemic mastocytosis: value of CD25, correlation with subvariants of the disease, and separation from mast cell hyperplasia. Am J Clin Pathol 2005;124:560-568.

7 Orfao A, Escribano L, Villarrubia J, et al. Flow cytometric analysis of mast cells from normal and pathological human bone marrow samples. Identification and enumeration. Am J Pathol 1996;149:1493-1499.

8 Escribano L, Orfao A, Diaz-Agustin B, et al. Indolent systemic mast cell disease in adults: immunophenotypic characterization of bone marrow mast cells and its diagnostic implications. Blood 1998;91:2731-2736.

9 Escribano L, Diaz-Agustin B, López A, et al. Immunophenotypic analysis of mast cells in mastocytosis: when and how to do it. Proposals of the Spanish Network on Mastocytosis (REMA). Cytometry B Clin Cytom 2004;58B:1-8.
10 Teodosio C, Garcia-Montero AC, Jara-Acevedo M, et al. Mast cells from different molecular and prognostic subtypes of systemic mastocytosis display distinct immunophenotypes. J Allergy Clin Immunol 2010;125: 719-726.

11 Sotlar K, Fridrich C, Mall A, et al. Detection of c-kit point mutation Asp-816-> Val in microdissected pooled single mast cells and leukemic cells in a patient with systemic mastocytosis and concomitant chronic myelomonocytic leukemia. Leuk Res 2002;26:979-984.

12 Garcia-Montero AC, Jara-Acevedo M, Teodosio C, et al. KIT mutation in mast cells and other bone marrow haematopoietic cell lineages in systemic mast cell disorders. A prospective study of the Spanish Network on Mastocytosis (REMA) in a series of 113 patients. Blood 2006;108:2366-2372.

13 Escribano L, Orfao A, Villarrubia J, et al. Immunophenotypic characterization of human bone marrow mast cells. A flow cytometric study of normal and pathological bone marrow samples. An Cell Pathol 1998;16:151-159.

14 Pardanani A, Kimlinger $\mathrm{T}$, Reeder $\mathrm{T}$, et al. Bone marrow mast cell immunophenotyping in adults with mast cell disease: a prospective study of 33 patients. Leuk Res 2004;28:777-783.

15 Bodni RA, Sapia S, Galeano A, et al. Indolent systemic mast cell disease: immunophenotypic characterization of bone marrow mast cells by flow cytometry. J Eur Acad Dermatol Venereol 2003;17:160-166.

16 Pardanani A, Kimlinger TK, Reeder TL, et al. Differential expression of CD2 on neoplastic mast cells in patients with systemic mast cell disease with and without an associated clonal haematological disorder. Br J Haematol 2003;120:691-694.

17 Escribano L, Orfao A, Villarrubia J, et al. Expression of lymphoid-associated antigens in mast cells: report of a case of systemic mast cell disease. Br J Haematol 1995;91:941-943.

18 Escribano L, Alvarez-Twose I, Sanchez-Munoz L, et al. Prognosis in adult indolent systemic mastocytosis: a long-term study of the Spanish Network on Mastocytosis in a series of 145 patients. J Allergy Clin Immunol 2009;124:514-521.

19 Alvarez-Twose I, Gonzalez de Olano D, SanchezMunoz L, et al. Clinical, biological and molecular characteristics of systemic mast cell disorders presenting with severe mediator-related symptoms. J Allergy Clin Immunol 2010;125:1269-1278.

20 Baumgartner C, Sonneck K, Krauth MT, et al. Immunohistochemical assessment of CD25 is equally sensitive and diagnostic in mastocytosis compared to flow cytometry. Eur J Clin Invest 2008;38:326-335.

21 Sanchez-Munoz L, Alvarez-Twose I, Garcia-Montero AC, et al. Evaluation of the WHO criteria for the classification of patients with mastocytosis. Mod Pathol 2011.

22 Caruso A, Licenziati S, Corulli M, et al. Flow cytometric analysis of activation markers on stimulated $\mathrm{T}$ cells and their correlation with cell proliferation. Cytometry 1997;27:71-76. 